\title{
Experimental characterization of emittance growth induced by the nonuniform transverse laser distribution in a photoinjector
}

\author{
F. Zhou, ${ }^{1,2}$ I. Ben-Zvi, ${ }^{1}$ M. Babzien, ${ }^{1}$ X. Y. Chang, ${ }^{1}$ A. Doyuran, ${ }^{1}$ R. Malone,${ }^{1}$ X. J. Wang, ${ }^{1}$ and V. Yakimenko ${ }^{1}$ \\ ${ }^{1}$ Accelerator Test Facility, Brookhaven National Laboratory, Upton, New York 11973 \\ ${ }^{2}$ Department of Physics and Astronomy, University of California at Los Angeles, Los Angeles, California 90095
}

(Received 25 February 2002; published 27 September 2002)

\begin{abstract}
The emittance of a high-brightness electron beam from a photoinjector is affected by the transverse and longitudinal distributions of the laser beam illuminating the cathode. A nonuniform laser beam generates a nonuniform electron-beam distribution that experiences emittance growth on a time scale of the plasma period. Experiments were performed at the Brookhaven Accelerator Test Facility to investigate the emittance growth due to transversely nonuniform laser beams. Laser masks were fabricated to generate various laser distributions. Significant emittance growth was observed as the laser distribution deviated strongly from a uniform distribution. For cylindrically symmetric, nonuniform distributions, experimental results agree with PARMELA simulations. The emittance dependence on the bunch charge is linear as a function of the bunch charge for both uniform and nonuniform beams. For a uniform beam, the emittance measurements agree well with the predictions from PARMELA simulations, but the analytical approach overestimates the results. For nonuniform beams, analytical estimates are about $70 \%$ of the measurements. For noncylindrically symmetric, nonuniform beams, we observed that the emittance is linearly proportional to the rms laser nonuniformity and the best emittance for a perfectly uniform beam is extrapolated to be $1.07 \pm 0.13 \mathrm{~mm}$ mrad at $0.5 \mathrm{nC}$.
\end{abstract}

DOI: 10.1103/PhysRevSTAB.5.094203

PACS numbers: 29.27.Bd, 41.75.Ht, 41.85.Ja

\section{INTRODUCTION}

High-brightness electron beams are critical for many applications, from $\mathrm{x}$-ray free electron lasers to electron cooling for high-energy colliders [1-3]. The highest brightness beams are produced by "photoinjectors" [4], that is microwave cavity-based electron guns in which the electrons are generated by photoemission from a cathode illuminated by a laser. The emittance of the electron beam in the photoinjector is mostly affected by three contributions: thermal emittance, radio frequency (rf) transverse kick induced emittance, and space-charge force induced emittance. The photoinjector's total emittance, projected for the whole bunch, can be approximated by

$$
\varepsilon_{n x}=\left[\left(\varepsilon_{n x}^{\mathrm{th}}\right)^{2}+\left(\varepsilon_{n x}^{\mathrm{rf}}\right)^{2}+\left(\varepsilon_{n x}^{\mathrm{sp}}\right)^{2}\right]^{1 / 2},
$$

where $\varepsilon_{n x}^{\text {th }}$ is the thermal emittance, $\varepsilon_{n x}^{\text {rf }}$ is the rf defocusing induced emittance, and $\varepsilon_{n x}^{\mathrm{sp}}$ is the space-charge induced emittance. This expression does not take into account various correlations between terms. The thermal emittance is related to the thermal energy of the photoemitted electrons by the expression, $\varepsilon_{n x}^{\text {th }}=\frac{r}{2} \sqrt{E_{\mathrm{th}} / m c^{2}}$, where $r$ is the transverse beam size on the cathode, $m c^{2}$ is the rest energy of the electrons, and $E_{\mathrm{th}}$ is the thermal energy of electrons. Without correlation between terms, the radially defocusing $\mathrm{rf}$ force induced emittance is proportional to the electric field strength in the cavity. The beam can be focused radially by magnetic focusing (e.g., a solenoid) which can reduce the projected emittance by removing longitudinally correlated bunch distortions. Space-charge effects, comprising linear and nonlinear forces, usually dominate the total beam emittance. While the linear space-charge forces can easily be compensated by solenoid focusing $[5,6]$, this does not hold for the nonlinear space-charge forces. Nonuniformity of the space-charge distribution results in nonlinear space-charge forces. There are two major sources of nonuniformity: quantum efficiency (QE) variation on the photocathode and laser illumination nonuniformity. In our experimental work, we produce a highly uniform quantum efficiency using a vacuum-based laser cleaning technique [7], and introduce an artificial, controlled nonuniformity of the laser illumination. In this paper we present experimental studies of the dependence of the emittance on uniformity and comparisons with PARMELA simulations and analytical estimations. Two types of nonuniformities are introduced: cylindrically symmetric and noncylindrically symmetric distributions.

\section{EXPERIMENTAL SETUP}

The experimental work was carried out at the Brookhaven Accelerator Test Facility (ATF). The electrons are produced at the ATF by a photoinjector, whose photocathode is illuminated by a frequency-quadrupled Nd:YAG laser. Two $S$-band (2856 MHz) linac sections are used to accelerate electrons. As mentioned in the introduction, the photocathode's QE is highly uniform because of a careful cleaning procedure. In routine operations, the Nd:YAG laser transverse distribution is nearly uniform. The nonuniform electron beams are produced by introduction of an artificially nonuniform laser beam by using special laser masks. 


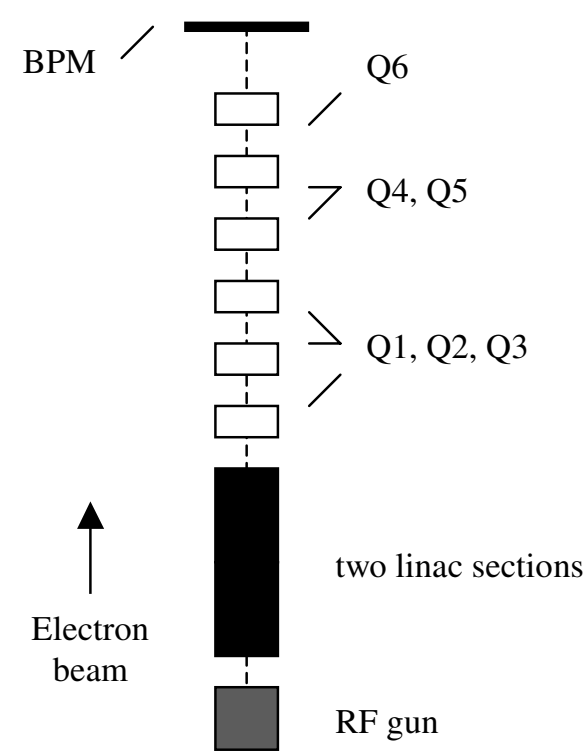

FIG. 1. Schematic layout for emittance measurement.

The transverse emittance is measured at a beam profile monitor (BPM) by scanning a quadrupole, Q6, as shown in Fig. 1, where the electron beam is tuned to $40 \mathrm{MeV}$. The BPM comprises a phosphorescent screen and a charge coupled device (CCD) camera with a frame grabber to record data. The detailed description for the measurement technique can be found in Ref. [6].

An ultrasmall electron-beam emittance has been achieved at the ATF $[4,8,16]$. The emittance is strongly dependent on the rf gun phase, and the minimum emittance can be achieved at a low rf gun phase [8]. These studies show that the space-charge force dominates the emittance at lower values of the laser-rf gun phase, but the rf defocusing forces dominate it at large phases. In our measurements, a lower gun phase $\left(30^{\circ}\right)$ is chosen. The variation of a photoelectron beam charge can be easily achieved by adjusting the laser energy. In other words, as the only variable parameters in the series of measurements described below are the nonuniform masks and the laser energy.

\section{EXPERIMENTAL RESULTS AND ANALYSIS}

\section{A. Cylindrically symmetric nonuniform laser}

The regular laser image can be measured by a CCD camera. It is shown that the beam is highly azimuthally symmetric. The spot size is about $2.3 \mathrm{~mm}$ in diameter. The transverse distribution of a beam slice taken along the beam diameter is measured, as shown in Fig. 2(red curve in the first plot). We observed that the peak-to-peak fluctuations relative to the flattop is less than $20 \%$ for this regular laser beam. For simplicity, we denote the various distributions by a "type" number. The original, unperturbed laser distribution is assigned to type 1 . Figure 2 also shows the sample distributions used for simulation (in blue) and analysis (in black). We started by producing and measuring cylindrically symmetric, nonuniform beams. This allows us to compare the experimental results with predictions from PARMELA [9] simulations and simple analytical analysis. Using laser masks, four types of cylindrically symmetric laser beams with different nonuniformities are produced. The transverse distribution of a beam slice taken along the beam diameter is measured for each nonuniform distribution, as shown in Fig. 2 (red curves). The transverse beam slice intensity distributions for various azimuthal angles are reasonably similar. In laser distribution type 2, a $45 \%$ peak-to-peak variation in laser intensity was introduced. For type 3, the center's intensity is about $65 \%$ of the outer ring. For type 4 , we introduced a peak-to-peak variation of about $75 \%$. Finally, in type 5, the middle part is blocked and thus the beam is significantly distorted compared with a regular uniform beam. The normalized transverse emittances of these distributions, shown in Fig. 3, are measured for a bunch charge of $0.48 \mathrm{nC}$. The emittance grows by about $30 \%$ for the $45 \%$ peak-to-peak variation, and by more than a factor of 2 for the extreme type 5 distribution. For each point, we took four to five measurements, and the data in the plot are the averaged values. The measurement error comes from the BPM resolution and from charge and phase jitters. The error due to the BPM resolution can be simply estimated as

$$
\delta \varepsilon=\frac{2 \sigma_{\text {res }}}{\sigma_{\text {beam }}} \varepsilon,
$$

where $\delta \varepsilon$ is the geometrical emittance error, $\sigma_{\text {res }}$ is the rms BPM resolution, $\sigma_{\text {beam }}$ is the measured $\mathrm{rms}$ beam size, and $\varepsilon$ is the geometrical beam emittance. The rms BPM resolution, $\sigma_{\text {res }}$, is about $25 \mu \mathrm{m}$. In the measurements, the rms electron-beam size $\sigma_{\text {beam }}$ at the BPM is about $250 \mu \mathrm{m}$ for the regular laser beam. Thus, the corresponding emittance measurement error is about $20 \%$. At the ATF, the laser beam is very stable: the timing jitter is smaller than $0.2 \mathrm{ps} \mathrm{rms}$ and the laser energy variation is smaller than $2 \%$ in rms [10]. Accordingly, the bunch charge jitter and phase jitter error is negligible as compared to the BPM resolution error. Using Eq. (2), we obtain error bars for each measurement point, as shown in Fig. 3. The PARMELA simulation results are compared with the measurements, as shown in Fig. 3. The profiles used for simulations shown in Fig. 2 (blue curve) are reasonably close to the measured profiles (red curves). The rms nonuniformity difference between the profile used for the simulation and the measured profile is on the order of $10 \%$ or less. According to the simulation, a $10 \%$ of rms nonuniformity variation in a profile will result in an emittance variation of $10 \%$.

In the simulations, we do not consider $\mathrm{QE}$ variation since the known QE variation of $10 \%$ peak to peak, 


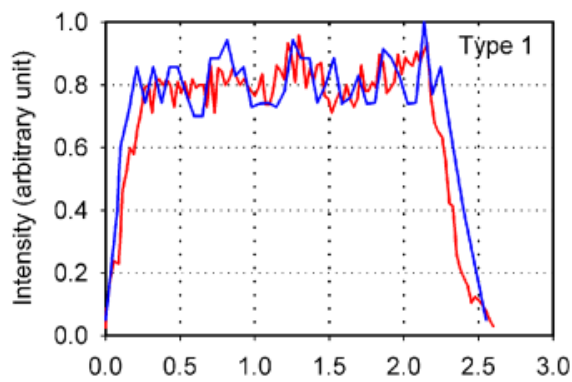

(a)

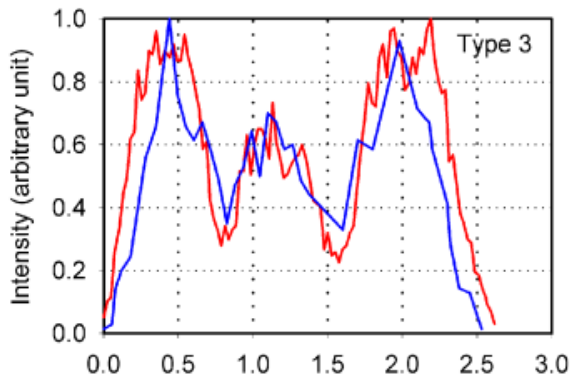

(c)

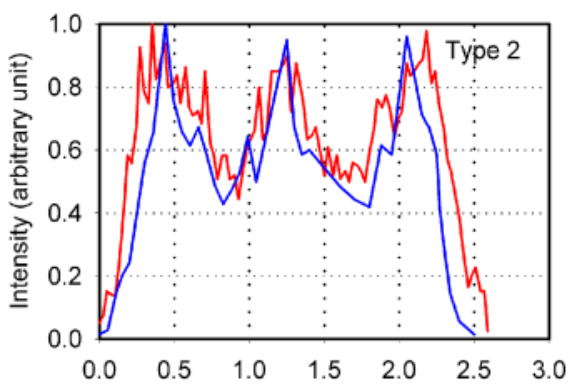

(b)

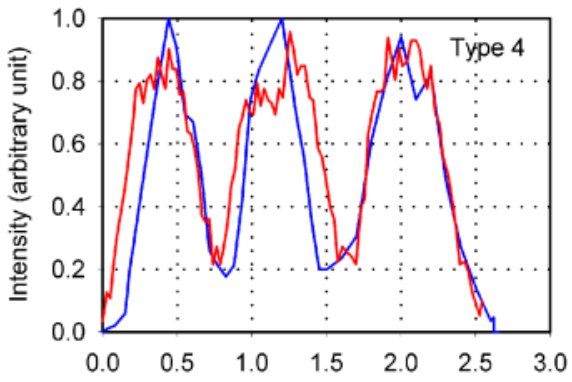

(d)

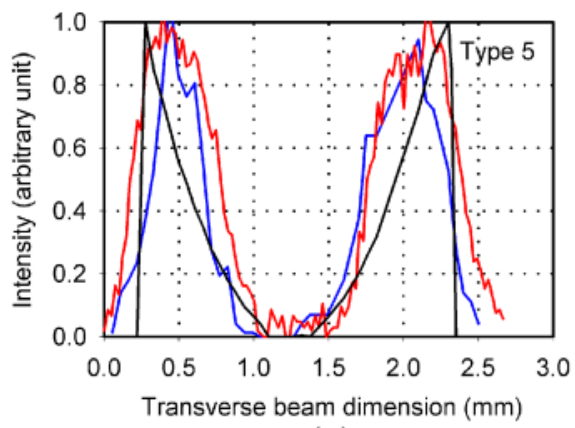

(e)

FIG. 2. (Color) Distribution of transverse laser beam slices along a beam diameter for the various distribution types. In addition to the measured data (in red), we show also the data used for simulations (in blue) or analysis (in black).

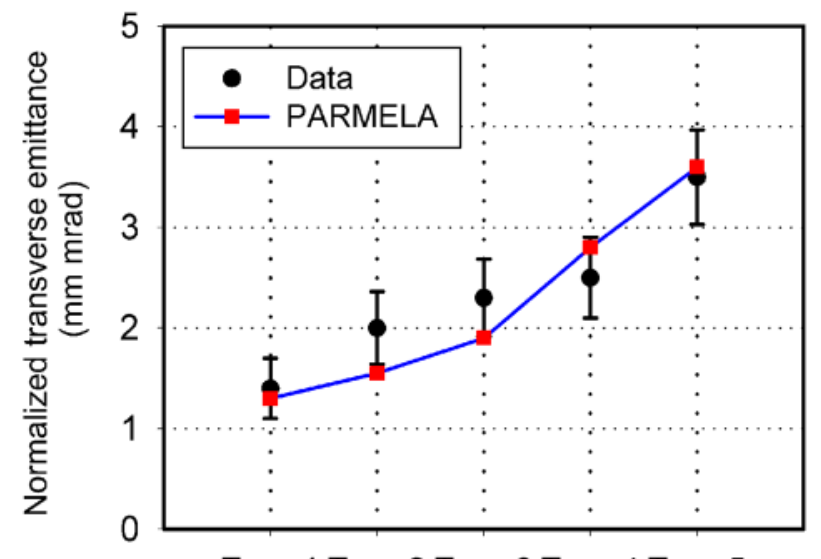

Type 1 Type 2 Type 3 Type 4 Type 5

\section{Laser type}

FIG. 3. (Color) Emittances versus laser distribution types. i.e., $<2 \% \mathrm{rms}$, is much smaller than the residual and artificially created laser nonuniformities. The sensitivity of the computed emittance was checked with respect to jitter from charge and phase. Emittance variations of 5\% and $2 \%$ are observed for $5 \%$ charge jitter and one-degree gun phase jitter, respectively. Next, the emittance dependence on the bunch charge for regular uniform and nonuniform beams with cylindrical symmetry was investigated experimentally. Experimental results as well as simulation are shown in Fig. 4. Laser distribution type 5 is taken as an example, being the most distorted distribution. The data show that the behavior of the emittance with bunch charge for both the uniform and nonuniform beams is similar, i.e., linear with the bunch charge. In addition to the PARMELA simulation, we provided an analytical estimate for comparison. The uniform beam can be considered as a charge cylinder [11], and the rf defocusing and 


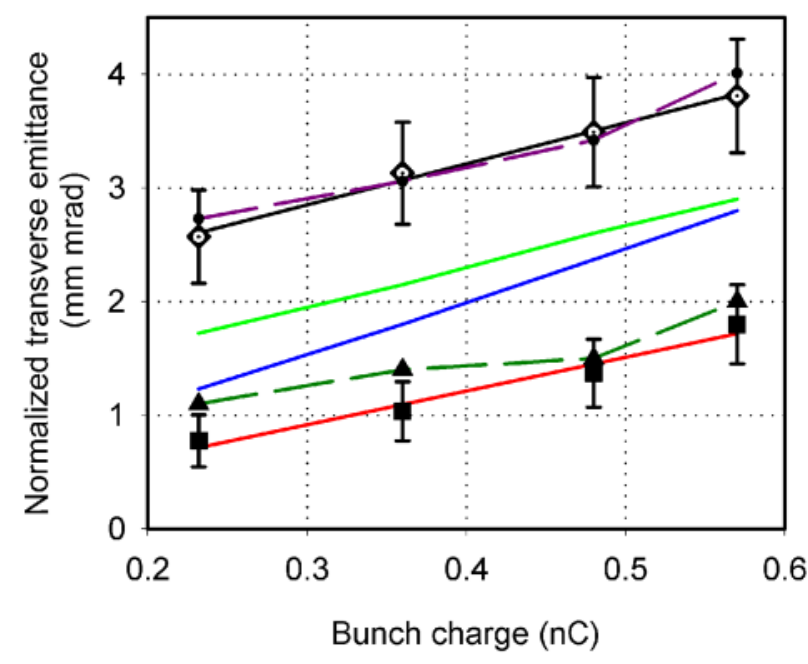

FIG. 4. (Color) Emittance as a function of bunch charge. Square markers indicate data for the regular beam; the red line shows best-fit data for a regular beam; the triangles with the dark green dotted line represent PARMELA simulations for a regular beam; the blue line is for an analytical estimate for the regular beam; the diamonds represent data for type 5; the black line is a best-fit curve for type 5; the circles with the dark pink dotted line show PARMELA simulation for type 5; the green line presents an analytical estimate for type 5 .

space-charge induced emittances, respectively, are derived as

$$
\begin{gathered}
\varepsilon_{n x}^{\mathrm{rf}}=\frac{\alpha r^{2} k^{3} \ell^{2}}{4 \sqrt{6} !}, \\
\varepsilon_{n x}^{\mathrm{sc}}=\frac{\pi}{4} \frac{1}{\alpha k} \frac{1}{\sin \phi_{0}} \frac{I}{I_{A}} \mu_{x}(A),
\end{gathered}
$$

where $\alpha=\left[\left(e E_{0}\right) /\left(2 m c^{2} k\right)\right], E_{0}$ is the maximum electric field on the cathode, $m c^{2}$ is the rest energy of the electrons, $k$ is the wave number, $r$ and $l$ are the radius and the length of the cylinder, respectively, $\phi_{0}$ is the rf phase as the particle leaves the cathode surface $z=0$ at $t=0, I$ is the peak current, $I_{A}=17 \mathrm{kA}$, and $\mu_{x}$ refers to the transverse space-charge factors. We used the ATF beam parameters (cathode electric field, beam size, and laser pulse length) and the rf defocusing-kick induced emittance is negligible. The total emittance is dominated by the space charge. Figure 4 shows this analytical approach overestimates the measured and simulated emittance growth for the uniform beam. That is because it does not take into account the emittance compensation, but it is still useful to guide estimates of the emittance. This model does not provide estimation for a nonuniform beam. To allow for that, we used another approach to estimate the emittance growth component due to nonuniformity. The electron beam can be described by a stationary or quasistationary Maxwell-Boltzmann distribution and in the space-charge dominated regime its temperature-dependent Boltzmann profiles tend to be uniform [12]. In the equilibrium state, no forces can affect the emittance. Any deviation from the nearly uniform density profile of a space-charge dominated MaxwellBoltzmann distribution will cause emittance growth, which can be represented by the energy difference between a nonstationary and a stationary beam [12-14]:

$$
\frac{\varepsilon_{n f}}{\varepsilon_{n i}}=\left(1+\frac{N r_{c} \tilde{x}}{15 \sqrt{5} \gamma_{0} \varepsilon_{n i}^{2}} \frac{U}{w_{0}}\right)^{1 / 2},
$$

where $\varepsilon_{n i}, \varepsilon_{n f}$ are the initial and final normalized emittances, respectively, $N$ is the particle number in one bunch, $r_{c}$ is the classical electron radius, $\tilde{x}$ is the rms transverse dimension, and $U / w_{0}$ is the normalized field energy difference per unit length between the nonuniform and the uniform initial beam. For nonuniform laser type 5, which is similar to the hollow shape shown in type 5 (see Fig. 2), $U / w_{0}$ was estimated in Ref. [13]. Substituting the ATF beam parameters into Eq. (5), the emittances are estimated at different bunch charges to compare with both the simulations and measurements, as shown in Fig. 4. It is clear that the analytical estimates for type 5 are about $70 \%$ of the measured emittances. Note that the regular beam (type 1) is not perfectly uniform, but has a slight nonuniformity due to QE variation (peak to peak $<10 \%$, i.e., $<2 \% \mathrm{rms}$ ) and residual laser (peak to peak $<20 \%$, i.e., $\sim 7 \% \mathrm{rms}$ ). The total $\mathrm{rms}$ base nonuniformity is quadratic for rms residual laser nonuniformity and rms QE nonuniformity, about $7.3 \% \mathrm{rms}$, which is smaller in comparison to the induced nonuniformities.

\section{B. Noncylindrically symmetric, nonuniform laser}

In practice, we cannot expect the nonuniformity of the beam distribution to show cylindrical symmetry. On the contrary, we expect the nonuniformity to be fine grained and randomly distributed. In order to study the emittance growth we produced masks that create nonuniform laser distributions with noncylindrical symmetry. Six masks are mounted in one plate. The first mask is simply glass with no distortion and is defined a $100 \%$ laser transmission. As mentioned in the last section, the nonuniformity in this regular laser comes from laser residual nonuniformity ( $<20 \%$ peak to peak, i.e., $\sim 7 \% \mathrm{rms}$ ) and QE nonuniformity ( $<10 \%$ peak to peak, i.e., $<2 \% \mathrm{rms}$ ). The total nonuniformity in the regular laser is quadratic for both nonuniformities, i.e., $7.3 \% \mathrm{rms}$. The next five masks have a rectangular grid pattern in which alternate squares have a reduced transmission, which varies from mask to mask. This creates a nonuniformity that is completely characterized by the transmission through the mask, an easily measured quantity. Thus the highest nonuniformity is at about 50\% transmission, when every other square is completely blocked. Thus we have the following masks with the laser transmission of about $100 \%, 90 \%, 80 \%, 70 \%, 60 \%$, and $50 \%$. The total 
rms nonuniformity for the following masks with laser transmission of about $100 \%, 90 \%, 80 \%, 70 \%, 60 \%$, and $50 \%$ are $7.3 \%, 11.2 \%, 15 \%, 28 \%, 33 \%$, and $40 \%$, respectively, as shown in Fig. 5. Note that the rms QE variation and rms laser nonuniformity are added in quadrature to obtain the total rms nonuniformities. As these masks are alternated during the measurements, the total laser energy is adjusted in order to keep the bunch charge constant at $0.46 \mathrm{nC}$. The results are shown in Fig. 6. With the mask of $50 \%$ transmission (i.e., $40 \%$ rms nonuniformity), the emittance is increased by about a factor of 2 . For a slight laser modulation, e.g., 90\% transmission (i.e., $11.2 \% \mathrm{rms}$ nonuniformity), the emittance is increased by about $30 \%$. A linear weight fit [15] of emittance as a function of rms nonuniformity is applied to the data (the weight corresponds to error bars) and as seen in Fig. 6 . The fit curve with tolerance of the slope is given as follows:

$$
\varepsilon_{n, \mathrm{rms}}=(1.07 \pm 0.13)+(0.055 \pm 0.0054) \cdot U_{\mathrm{non}},
$$

where $\varepsilon_{n, \text { rms }}$ is the normalized emittance, and $U_{\text {non }}$ is the rms nonuniformity. As mentioned above, the regular laser is not perfectly uniform, but has $7.3 \%$ rms nonuniformity. This means that our best-measured emittance has some contribution from nonuniformity. Projected the emittance at zero of the nonuniformity in Eq. (6), the best

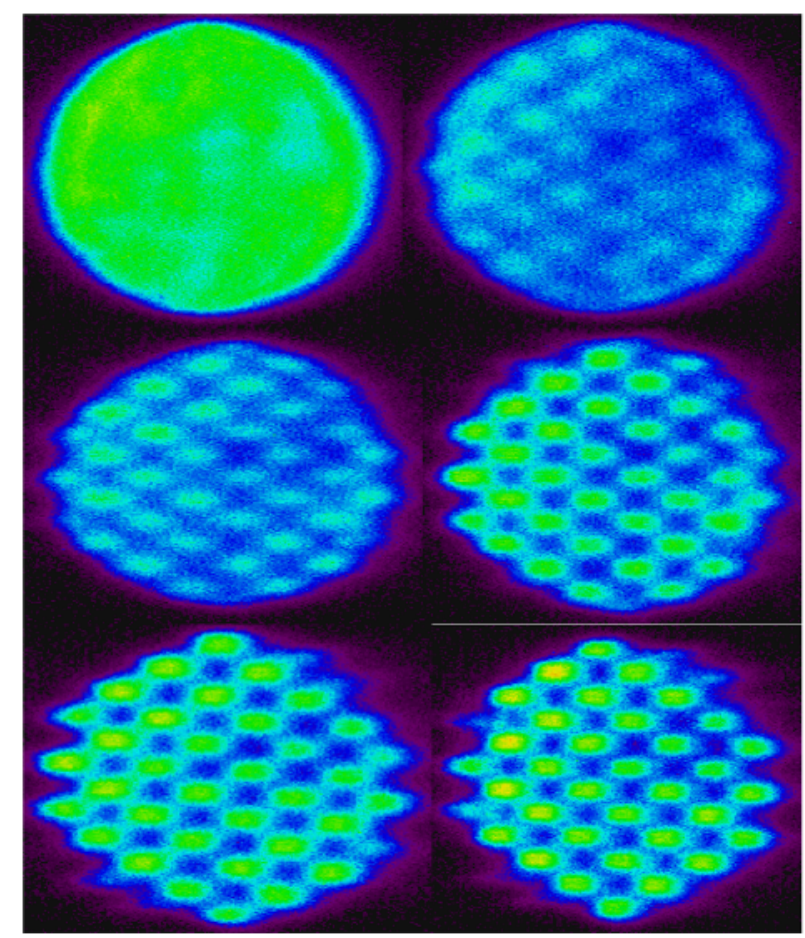

FIG. 5. (Color) Images of a noncylindrically symmetric laser with different rms nonuniformities. The rms nonuniformity for each image is calculated as follows. Top left: $7.3 \%$; top right: $11.2 \%$; middle left: $15 \%$; middle right: $28 \%$; bottom left: $33 \%$; bottom right: $40 \%$.

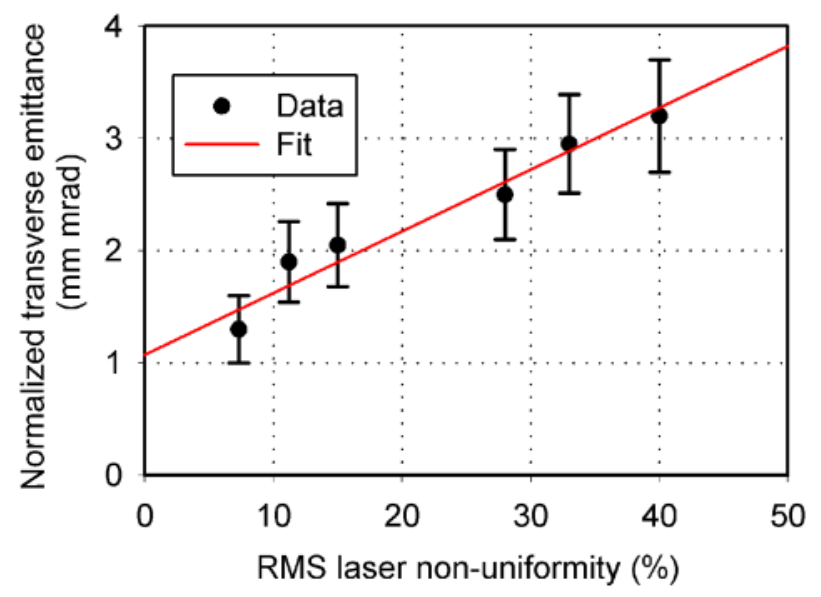

FIG. 6. (Color) Emittance as a function of laser rms nonuniformity.

emittance is $1.07 \pm 0.13 \mathrm{~mm}$ mrad. This value is slightly larger than the previous best-measured emittance of $0.8 \pm 0.12 \mu \mathrm{m}$ at the same charge at the ATF [16]. One should note that the conditions for the previous measurement were improved. One improvement is to employ a higher field in the rf gun. The higher electric field improves the emittance. According to our PARMELA simulations, a $10 \%$ higher field improves the emittance by $10 \%$. The beam trajectory in the ATF linac is strongly influenced by the first three quadrupoles downstream of the linac. In the previous measurements, we used beam-based alignment to place those quadrupoles on the same axis as the linac. This alignment of quadrupoles decreased beam distortions due to the wakes in the linac [16].

\section{SUMMARY}

We report experimental results and, where practical, comparison with numerical simulation and analytical estimates for the dependence of the transverse emittance of an electron beam on the nonuniformity of the charge distribution in the transverse plane. For cylindrically symmetric electron beams, we observe a linear dependence of the emittance on the bunch charge for the various degrees of nonuniformity. The emittance measurements agree well with simulations and show emittance growth by a factor of 2 for the highest nonuniformity. For the uniform beam, the emittance measurements agree well with the predictions from PARMELA simulations, but the analytical approach overestimates the emittances. For the most nonuniform beam, the analytical estimates are about $70 \%$ of the measured emittances.

For noncylindrically symmetric, nonuniform beams, the emittance is linearly proportional to the laser rms nonuniformity and the best emittance for the perfectly uniform beam is extrapolated to be $1.07 \pm 0.13 \mathrm{~mm} \mathrm{mrad}$ at $0.5 \mathrm{nC}$.

We conclude that the measurements agree with the predictions that emittance grows as a function of 
nonuniformity. Our results can provide a valuable guide to the estimating of the effect of laser nonuniformity in this critical range of very-high brightness electron beams. The results for a noncylindrical symmetry suggest that a theoretical model of small-grained nonuniformity may be developed but this task is beyond the scope of the present work.

\section{ACKNOWLEDGMENTS}

This work was done under the auspices of the U.S. Department of Energy Grant No. DE-AC02-98CH10886.

[1] LCLS Conceptual Design Report, SLAC, 2002.

[2] I. Ben-Zvi et al., in Proceedings of the PAC01, Chicago, IL, 2001 (IEEE, Piscataway, NJ, 2001), pp. 350-352.

[3] J. Andruszkow et al., Phys. Rev. Lett. 85, 3825 (2000).

[4] X. J. Wang, in Proceedings of the PACO1, Chicago, IL, 2001 (Ref. [2]), pp. 81-85.

[5] B. E. Carlsten, Nucl. Instrum. Methods Phys. Res., Sect. A 285, 313 (1989).
[6] X. Qiu et al., Phys. Rev. Lett. 76, 3723 (1996).

[7] X.J. Wang et al., in Proceedings of the LINAC98, Chicago, IL, 1998 (ANL Report No. ANL-98/28, ANL, 1998), pp. 866-868.

[8] X. J. Wang, X. Qiu, and I. Ben-Zvi, Phys. Rev. E 54, 3121 (1996).

[9] L. Young, PARMELA code manual, LANL Report No. LA-UR-96-1835, 1996.

[10] B. Babzien, ATF YAG laser system, ATF user meeting, 2001.

[11] K. J. Kim, Nucl. Instrum. Methods Phys. Res., Sect. A 275, 201 (1989).

[12] M. Reiser, Theory and Design of Charged Particle Beams (Wiley, New York, 1994), ISBN 0-471-30616-9.

[13] T. P. Wangler, F.W. Guy, and I. Hofmann, in Proceedings of the LINAC'86 (SLAC Report No. SLAC-R-303, SLAC, 1986), pp. 340-345.

[14] M. Reiser, AIP Conf. Proc. 193, 311 (1989).

[15] P. Bevington, Data Reduction and Error Analysis for the Physics Sciences (McGraw-Hill, New York, 1969).

[16] V. Yakimenko et al., Nucl. Instrum. Methods Phys. Res., Sect. A 483, 277 (2002). 